\title{
CONTRAST-SPECIFIC ULTRASONIC FLOW MEASUREMENTS BASED ON BOTH INPUT AND OUTPUT TIME INTENSITIES
}

\author{
Chin-Kuang Yeh,* MeI-Ju Yang ${ }^{*}$ and PaI-ChI Li* ${ }^{* \dagger}$ \\ *Department of Electrical Engineering, National Taiwan University, Taipei, Taiwan; and ${ }^{\dagger}$ Division of Medical \\ Engineering Research, National Health Research Institutes, Taipei, Taiwan
}

(Received 22 April 2002; in final form 22 November 2002)

\begin{abstract}
Ultrasonic contrast agents are used to assess perfusion conditions based on evaluation of the timeintensity curve. Such a curve reflects the concentration of microbubbles in the perfused area and the indicatordilution theory is used to derive the volumetric flow rate from the measured concentration. Previous results have shown that the technique is not reliable in some conditions due to the shadowing effect. To overcome this problem, a contrast-specific technique using both the input and output time-intensity relationships is proposed; this contrasts with conventional techniques that utilize only the relationship directly from the perfused area. The proposed technique is referred to as the input-output time-intensity curve (IOTIC) method. In this work, the shadowing effect was studied experimentally and the efficacy of the IOTIC technique was assessed and compared with conventional techniques. The results indicate that the IOTIC technique eliminates the shadowing effect and provides a good correlation between the actual flow rate and measured flow-related parameters; thus, making quantitative estimation of perfusion feasible. Note that the IOTIC is applicable, based on the assumption that both the input and the output can be positioned within the same image plane; its clinical applications include situations where the perfused area cannot be effectively imaged by ultrasound (US). One example is the assessment of brain perfusion, and it will be used as a target clinical application of the IOTIC technique. (E-mail: paichi@cc.ee.ntu.edu.tw) (C) 2003 World Federation for Ultrasound in Medicine \& Biology.
\end{abstract}

Key Words: Flow estimation, Indicator-dilution theory, Shadowing effect, Time-intensity curve, Ultrasonic contrast agent.

\section{INTRODUCTION}

Ultrasonic contrast agents have been used to enhance the acoustic backscattered intensity of blood and, thereby, assist the assessment of blood flow parameters. Several contrast-specific imaging techniques based on the indicator-dilution theory have been proposed (Claassen et al. 2001; Heidenreich et al. 1993; Li et al. 2002; Nanda et al. 1997; Ugolini et al. 2000; Wilkening et al. 1999). The indicator-dilution theory provides a mathematical model for estimating hemodynamic parameters using changes in the backscattered intensity as a function of time (i.e., the time-intensity curve, TIC). The TIC can be measured within the perfused area or it can be measured at the outflow of the perfused area when a bolus of contrast agent is injected at the inflow. In this case, a linear system model can be applied and the TIC then represents

Address correspondence to: Pai-Chi Li, Department of Electrical Engineering, National Taiwan University, No. 1, Sec. 4, Roosevelt Road, Taipei 106 Taiwan. E-mail: paichi@cc.ee.ntu.edu.tw the transfer function of the dilution process (Mor-Avi et al. 1993a, 1993b).

Several contrast-based techniques that measure the TICs within the perfused area have been proposed. Ugolini et al. (2000) performed absolute and relative flow quantification using harmonic power Doppler imaging, and they found a good correlation between the absolute flow and several flow parameters derived from the TIC. However, the power Doppler mode is unreliable due to potential motion artefacts; the flow velocities may be too slow to be distinguished from tissue motion. Simpson et al. (2001) proposed a Doppler filter technique using radiofrequency data to eliminate the motion artefacts.

Wilkening et al. (1999) compared three contrastbased imaging modes (i.e., contrast harmonic imaging, contrast burst imaging and time variance imaging) to detect the brain perfusion through the transtemporal bone window using Levovist ${ }^{\circledR}$. These three modes were designed to detect nonlinearities and microbubble destruction. However, the high attenuation of the transtemporal 
bone window makes visualization of brain perfusion unreliable, even at high acoustic powers.

Quantification of perfusion has also been investigated using harmonic grey-scale imaging in a capillary flow model (Claassen et al. 2001). They found that the indicator-dilution theory is not applicable for perfusion analysis, possibly due to the effect of bubble saturation. Bos et al. (1996) also indicated that depth-dependent attenuation (i.e., shadowing effect) affects perfusion measurement. Postert et al. (2000) used different techniques to visualize cerebral contrast enhancement in different brain areas. All quantitative data of that study revealed large variations between individuals with different blood pressure, heart rate and depth-dependent attenuation. The study showed that the shadowing effect occurred when the mean distance between the microbubbles became relatively small.

Instead of direct measurements within a perfused area, some studies have measured the TICs at the outflow of the perfused area when a bolus of contrast agent is injected at the inflow. Schwarz et al. (1993) used the outflow TICs obtained from ultrasonic radiofrequency, video and Doppler data to assess flow parameters. The results showed a linear relationship between the washout rate and the volumetric flow rate. Chen et al. (1998) provided a mathematical model for estimating hemodynamic parameters based on the indicator-dilution theory. Li et al. (2002) found that several factors need to be considered for quantitative flow analysis. For example, the microbubbles may not be completely mixed when a large mixing chamber is used, so that only relative flow quantification can be achieved and absolute flow quantification is not possible unless the effective mixing volume can be found. In addition, the relative inflow/outflow directions also affect the derived time constants.

Variations in the concentration of the contrast agent with time can be determined mathematically by modeling the flow system as a series of blood-mixing chambers (Li et al. 2002; Yeh et al. 2001). However, these timeintensity-based methods are not as effective when the injection site is far away from the measurement site, in which case the time-intensity relationship at the inflow must also be taken into account. If the blood-mixing chamber can be treated as a linear time-invariant system, it may be possible to apply deconvolution techniques for flow estimation if the time-intensity relationships are measured at both the input and the output of the bloodmixing chamber (Yeh et al. 2001). Our previous results showed that, although the linear time-invariant assumption may not be valid, time-intensity-based methods are still feasible if only relative flow analysis is required.

In the present study, an in vitro perfusion model was set up to measure three TICs (inflow, outflow and the perfused area) simultaneously using grey-scale imaging.
One purpose of this study was to evaluate the effect of shadowing on TIC-based perfusion assessment. In the perfused area, shadowing was evaluated at different regions-of-interest (ROIs) during different flow rates. The correlation between flow parameters and the various TICs is also discussed. Moreover, a new technique is proposed in this study for perfusion estimation based on both the inflow and the outflow TICs, and is referred to as the input-output TIC (IOTIC) method. The TIC based on the IOTIC method is compared to the TIC measured within the perfused area. The relationships between flow parameters and parameters of the IOTICs are also discussed. Note that the injection site can be far away from where the inflow TIC is measured, because both input and output time intensities are taken into account.

Note that the proposed IOTIC technique is applicable only when both the input TIC and the output TIC can be reliably measured. In addition, such a technique is more suitable when the perfusion is homogeneous. Nonetheless, the IOTIC technique may have other potential benefits. One example is to apply deconvolution techniques to estimate the transfer function of the dilution process in the perfused area. With such information, it may be possible to correlate the estimated transfer function with local perfusion conditions. The primary purpose of the current study was to test the hypothesis that the IOTIC can be used to avoid the shadowing effect. The long-term research goal was to assess brain perfusion by measuring the time-intensity relationship in the extracranial flow. Specifically, the time-intensity curves were measured both in a carotid artery and a jugular vein. Therefore, the shadowing effect and the poor acoustic window can be avoided because the TIC from the brain tissue is no longer needed.

\section{MATERIALS AND METHODS}

\section{Basic principles}

Perfusion is defined as the flow per unit volume (Nanda et al. 1997). When a contrast agent is injected, the perfusion can be calculated by dividing the fractional vascular volume by the mean transit time (MTT). In other words, if the TIC and vascular volume in the ROI can be measured, perfusion can be calculated. Because perfusion represents blood flow normalized to the quantity of tissue supplied, it is clearly a very important measure of the microcirculation (Goldberg 1997; Nanda et al. 1997).

Considering the simple perfusion model depicted in Fig. 1, blood flow at a rate $(Q)$ enters and leaves a perfusion model of volume $(V)$ via a single input vessel and a single output vessel. The concentration of contrast agent entering the model (i.e., the number of microbubbles per unit volume) as a function of time (i.e., the 


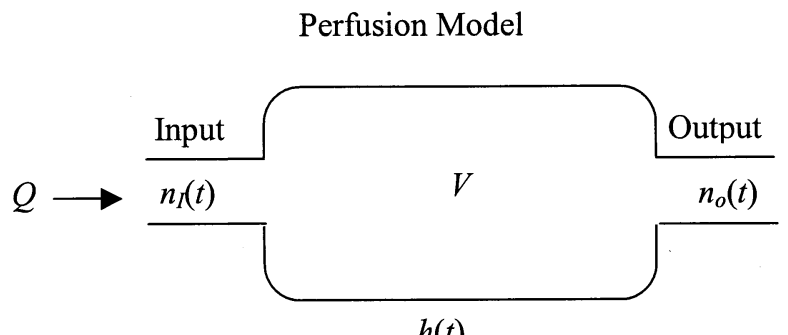

Fig. 1. A schematic diagram of the perfusion model.

input function) is defined as $n_{I}(t)$. According to the dilution theory, the concentration of the contrast agent at the output end of the chamber $n_{o}(t)$ (i.e., the output function) is given by:

$$
n_{o}(t)=n_{I}(t) \otimes h(t),
$$

where $\otimes$ stands for convolution and $h(t)$ is the transfer function of the perfusion model. Note that the measured intensity is assumed to be linearly related to the concentration; this assumption has also been made in other studies (Heidenreich et al. 1993; Mor-Avi et al. 1993a, 1993b; Li et al. 2002; Schwarz et al. 1993; Yeh et al. 2001).

The time constant $\tau$, which can be considered as the system's transit time, is defined as the ratio of the mixing volume to the volume flow rate (i.e., $\tau=V / Q$ ). In this case, the transfer function is given by (Chen et al. 1998):

$$
h(t)=\left\{\begin{array}{cc}
0 & t<0 \\
\frac{1}{\tau} e^{-t / \tau} & t>0
\end{array}\right.
$$

In addition to $\tau$, the TIC is used to derive other parameters in this study, including MTT and area under the curve (AUC). The MTT is defined as:

$$
M T T=\frac{\int_{0}^{\alpha} t f(t) d t}{\int_{0}^{\alpha} f(t)},
$$

where $f(t)$ is the measured intensity as a function of time $t$. The MTT represents the time for the entire fluid volume to pass though the perfused area. According to the indicator-dilution theory, there is an inverse relation between the flow rate and the $M T T$. If the transfer function is defined as in (2), it can be shown that:

$$
M T T=\tau=\frac{V}{Q}
$$

In the experiments of this study, the flow rate $Q$ ranged from 700 to $1300 \mathrm{~mL} / \mathrm{min}$. The area under the curve (AUC) is defined as:

$$
A U C=\int_{0}^{\infty} f(t) d t
$$

It was shown in Claassen et al. (2001) that the AUC is also directly related to the perfusion blood volume. It may be affected by the multipath acoustic scattering, and can also be used to test the linear relationship between the concentration and the backscattered intensity if the time constant is relatively short compared to the total measurement time (Yeh et al. 2001).

To reduce estimation errors in the time-intensity measurements, the TIC is fitted to a gamma function $g(t)$ defined by:

$$
g(t)=\alpha\left(t-t_{o}\right)^{\gamma} e^{-\beta\left(t-t_{0}\right)},
$$

where $t_{0}$ indicates a delay time, $\alpha$ and $\beta$ are scaling factors, and $\gamma$ represents the skewness. The gamma function is used to represent a wide range of characteristics of possible TIC curves (Chen et al. 1998). In this study, all flow-related parameters were calculated after gammafunction fitting. Certainly, the validity of the gammafunction fitting may be questionable if the flow model cannot be adequately described by the indicator-dilution theory. The correlation coefficients between the original time-intensity curves and the fitted gamma function must be higher than 0.9 .

\section{Input-output time-intensity curve technique}

The proposed IOTIC technique monitors the acoustic intensities at both the inflow and the outflow of a perfused region. Based on mass conservation, the difference between the number of microbubbles at the output and at the input at a time $t$ must be the same as the time-derivative of the number of microbubbles in the perfusion model, $n(t)$, (Cooney 1976; Li et al. 2002; Miles et al. 1997). Thus, assuming a constant volume flow rate $Q$, the number of microbubbles entering and leaving the mixing chamber at time $t$ is $Q \times n_{I}(t)$ and $Q$ $\times n_{o}(t)$, respectively. It can then be shown that:

$$
V \frac{d n(t)}{d t}=Q n_{I}(t)-Q n_{o}(t)=Q\left(n_{I}(t)-n_{o}(t)\right) .
$$




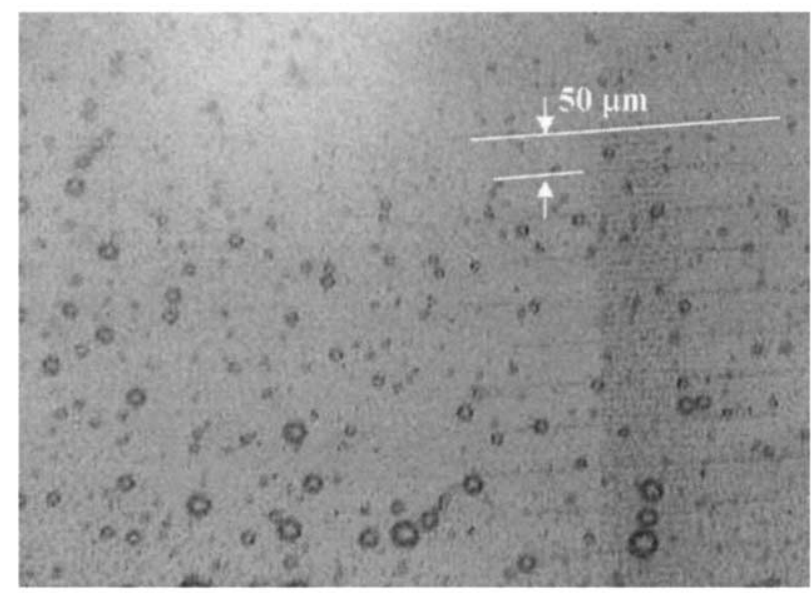

Fig. 2. Photograph of the sonicated albumin-based contrast agent.

By integrating both sides of eqn (7), we have:

$$
n(t)=\frac{Q}{V} \int_{0}^{t}\left(n_{I}\left(t^{\prime}\right)-n_{o}\left(t^{\prime}\right)\right) d t^{\prime} .
$$

In other words, it is possible to evaluate the concentration in the perfused area by simultaneously measuring the TICs at the input and output ends. Moreover, $n(t)$ is inherently less noisy due to the integration term in eqn (8).

\section{Contrast agent}

The contrast agent used in this study was self-made sonicated albumin. The procedure used to produce the agent was similar to that described by Bos et al. (1996). To heat the solution, $8 \mathrm{~mL}$ of $25 \%$ human albumin (Octapharma, Vienna, Austria) was sonicated by a sonicator at a low-amplitude setting of $40 \%$ (model 450, Branson Ultrasonics Corporation, Danbury, CT). After $60 \mathrm{~s}$, the acoustic amplitude was increased to $60 \%$ to sonicate the albumin for another $60 \mathrm{~s}$. Stable microbubbles were formed by surface agitation. The sonicator was switched off and another $8 \mathrm{~mL}$ of albumin was administered at room temperature. The solution was stirred carefully and $3 \mathrm{~mL}$ of contrast agent was withdrawn from beneath the foamy surface. The diameter of the microbubbles was measured under a light microscope (model EMZ-TR, Meiji, Tokyo, Japan), and ranged from 5 to $20 \mu \mathrm{m}$. Figure 2 shows an image of the albuminbased contrast agent. Note that the bubbles may appear to be artificially large in the image because they were flattened when taking the picture. The size distribution of the contrast agent was measured by a particle size analyzer (LS 230, Beckman Coulter, Fullerton, CA). The median diameter was $18 \mu \mathrm{m}$ and the SD was $5 \mu \mathrm{m}$. On the other hand, the attenuation as a function of frequency was measured by the insertion-loss technique (Chang and Shung 1993). The attenuation was $25 \mathrm{~dB} / \mathrm{cm}, 19$ $\mathrm{dB} / \mathrm{cm}$ and $16 \mathrm{~dB} / \mathrm{cm}$ at $2 \mathrm{MHz}, 3 \mathrm{MHz}$ and $4 \mathrm{MHz}$, respectively.

\section{Experimental setup}

The perfusion phantom was made from a renal dialysis cartridge (model C-12, Terumo, Tokyo, Japan). A window was made in the surface of the cartridge casing, which was then wrapped in a membrane to avoid microbubble leakage into the surrounding water tank. The cartridge consisted of 8600 hydrophilic capillaries, each with a mean diameter of $200 \mu \mathrm{m}$ and a length of $235 \mathrm{~mm}$, making a total capillary volume of $79 \mathrm{~mL}$. An additional sphere was inserted between the injection site and the perfusion phantom, to simulate the situations where the injection site is far away from the measurement site (Yeh et al. 2001). Two 8-mm diameter silicone tubes (with opposite flow directions) and the perfusion phantom were fixed in parallel and immersed in a water tank. A curved-array transducer (model C4-2, Advanced Technology Laboratories, Bothell, WA) was placed 1.5 $\mathrm{cm}$ above the cartridge dialysis. A damper was used to stabilize the flow. To prevent recirculation of the contrast agent back in the phantom, the water only passed through the model once. A schematic diagram of the configuration is shown in Fig. 3, and the markers A, B, and C represent the ROI positions of the input, the perfused area and the output, respectively.

\section{Image processing}

The time-intensity relationships at the input, the output and the perfused area were measured simultaneously using a commercial US device (Ultramark 9, Advanced Technology Laboratories). The mechanical index was 0.7 , with the frame rate ranging from 20-30 Hz. Grey-scale images of the transverse view of the three ROIs were acquired at the rate of one frame per s. A total of 300 image frames (i.e., 5 min of sampling) were acquired and digitized by a frame grabber (model UPG401B, Upmost, Taipei, Taiwan) and stored for offline analysis. The image size of $304 \times 232$ pixels covered an area of $72 \times 55 \mathrm{~mm}$. A typical image is shown in Fig. 4. The three ROIs denoted by the three circles correspond, from right to left, to the input tube, the dialysis cartridge (i.e., the perfused area), and the output tube. The B-mode dynamic range was $50 \mathrm{~dB}$. The greyscale image data were converted from a logarithmic to a linear scale, before the mean backscattered intensities inside the ROIs were calculated for the time intensities. 


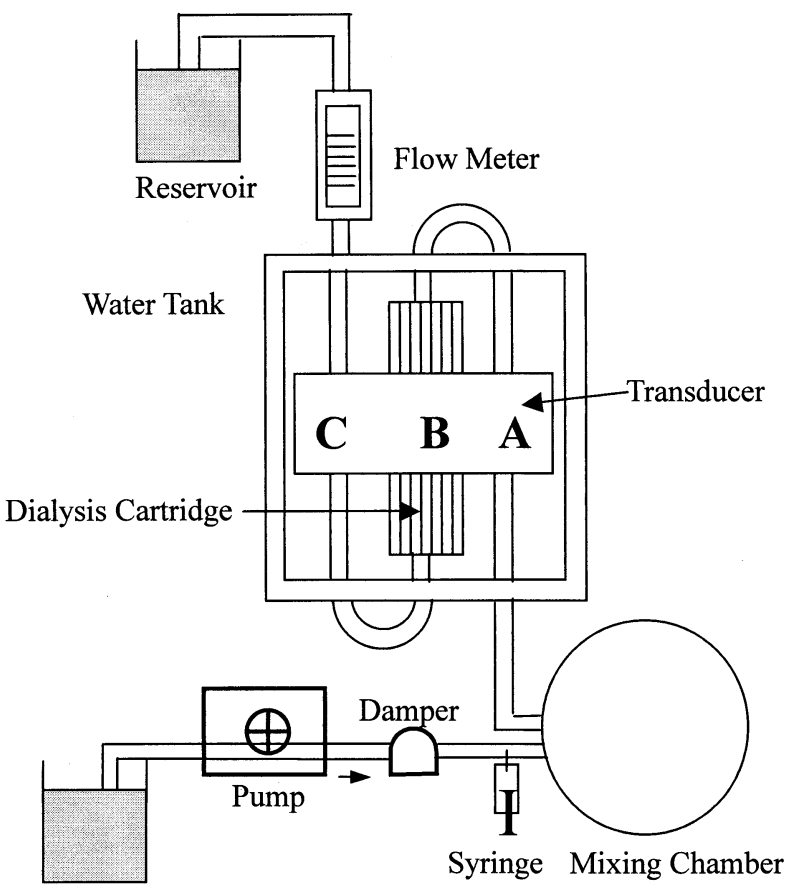

Reservoir

Fig. 3. Experimental setup with a perfusion phantom.

To obtain the TICs, the inner portions of the tubes were covered by circles of different diameters. The dialysis cartridge, on the other hand, was covered by half rings of different widths. The intensities of the pixels inside the ROIs were summed and the resulting value was used as the intensity at that particular instance. The procedure continued until all the 300 digitized grey-scale images were processed.

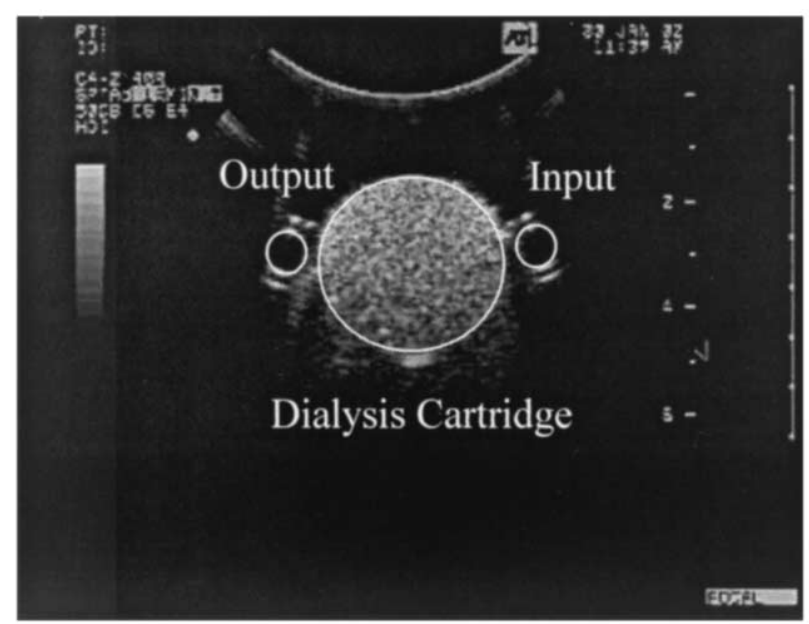

Fig. 4. A typical grey-scale image with three ROIs indicated.
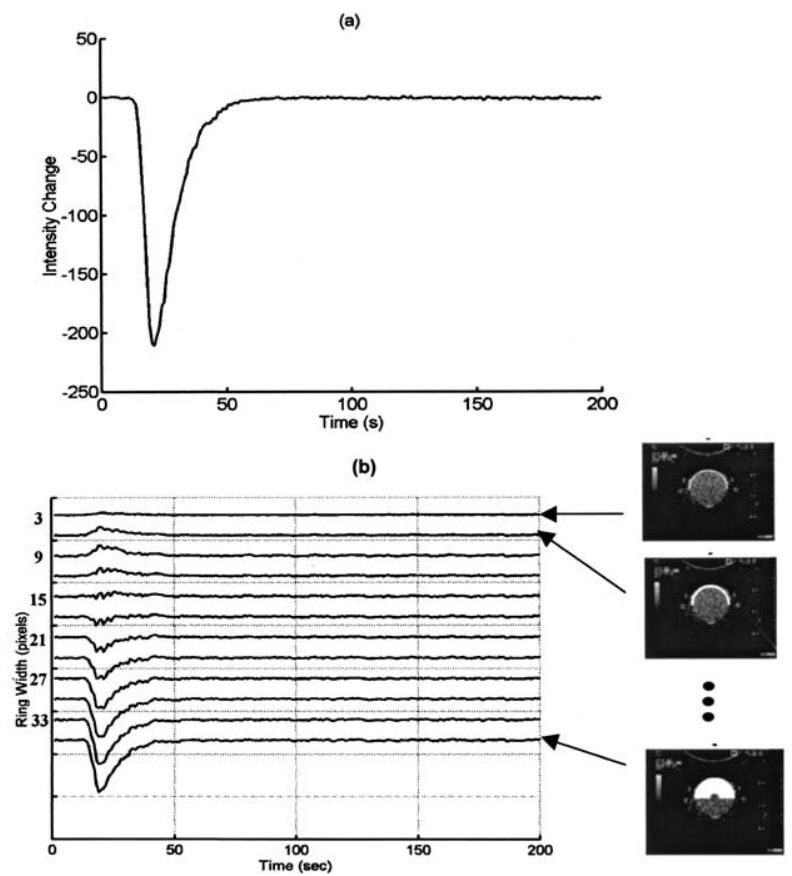

Fig. 5. Time-intensity curves (TICs) are obtained using (a) a circle as the ROI, and (b) half-rings with different widths as ROIs. The flow rate was $900 \mathrm{~mL} / \mathrm{min}$.

\section{EXPERIMENTAL RESULTS}

\section{Shadowing effect}

Experiments were conducted to evaluate the influence of the shadowing effect on the perfusion estimates. Four flow rates of 700, 900, 1100 and $1300 \mathrm{~mL} / \mathrm{min}$ were used. For each flow rate, five independent measurements were performed to obtain the mean and the SD. A typical TIC is shown in Fig. 5a, for which the flow rate was 900 $\mathrm{mL} / \mathrm{min}$. It can be seen that the measured intensity decreased as the microbubbles passed through the perfusion phantom. The same effect occurred during other flow rates; this was due to the shadowing effect induced by the microbubbles. The microbubbles were close together in the perfused area and multipath reverberations reduced the backscattered signal. Note that relative intensity change is shown in Fig. 5a. In other words, the backscattered intensity before microbubbles entered the dialysis cartridge was used as the baseline. As microbubbles passed through the capillaries, the shadowing effect occurred and it made the summed intensity negative.

To explore the shadowing effect further, various ROIs were used. The ROIs were half-rings with various widths covering the upper portion of the dialysis cartridge. The TICs corresponding to different degrees of shadowing effect can be obtained by changing the ring width (in number of pixels) of the specific ROI. Figure 
(a)

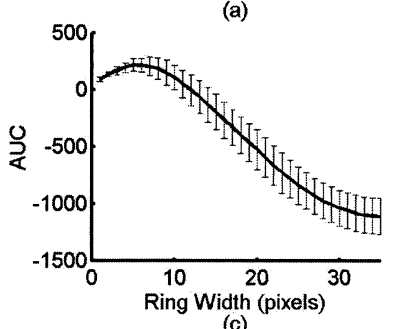

(c)

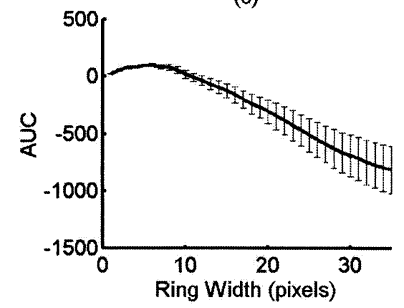

(b)

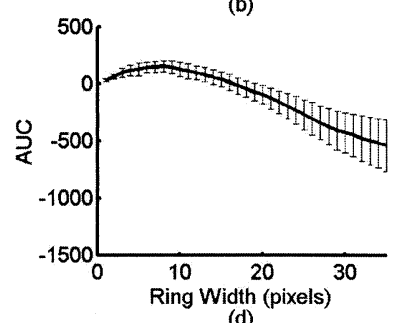

(d)

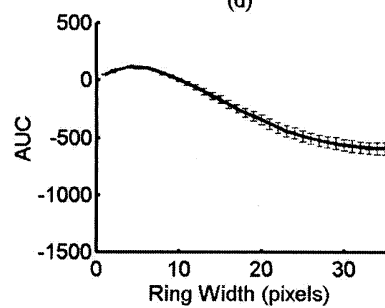

Fig. 6. AUC of the TIC vs. ROI width at flow rates of (a) 700, (b) 900 , (c) 1100 and (d) $1300 \mathrm{~mL} / \mathrm{min}$.

$5 \mathrm{~b}$ displays the TICs for various ring widths; the ring width increased from top to bottom, with a step size of three pixels between each curve. The upper TIC is for a ring width of three pixels; in this case, the intensity increased as the contrast agent passed through the ROI. As the ring width increased, the intensity became smaller as the agent passed through the ROI. These results indicate that the shadowing effect depends on the size and position of the ROI and, hence, illustrates the importance of defining the ROI correctly in studies of this type.

Figure 6 demonstrates the relationship between the AUC and the ROI ring width. Figure $6 \mathrm{a}-\mathrm{d}$ shows the results at flow rates of 700,900, 1100 and $1300 \mathrm{~mL} / \mathrm{min}$, respectively. The horizontal axis represents the ring width (in number of pixels) and the vertical axis is the AUC of the TIC. The average values are shown, with the error bars representing $\pm 1 \mathrm{SD}$. Similar to Fig. 5, relative AUC changes are shown and, thus, the values become negative when the shadowing effect is significant. The results show that the AUC varies with the ring width. The AUC values increased for ring widths up to fivenine pixels, and decreased for larger values. The shadowing effect becomes significant as the width of the ROI center increases. The maximum AUC illustrates that the TIC suffered the least shadowing effect. The results also demonstrate that the shadowing effect is related to flow rate.

\section{Input-output time-intensity curve technique}

Figure 7a shows typical TICs from experimental data. A flow rate of $900 \mathrm{~mL} / \mathrm{min}$ was used. The input, the output and the perfusion TICs directly measured from raw grey-scale images are shown as the dashed, dotted
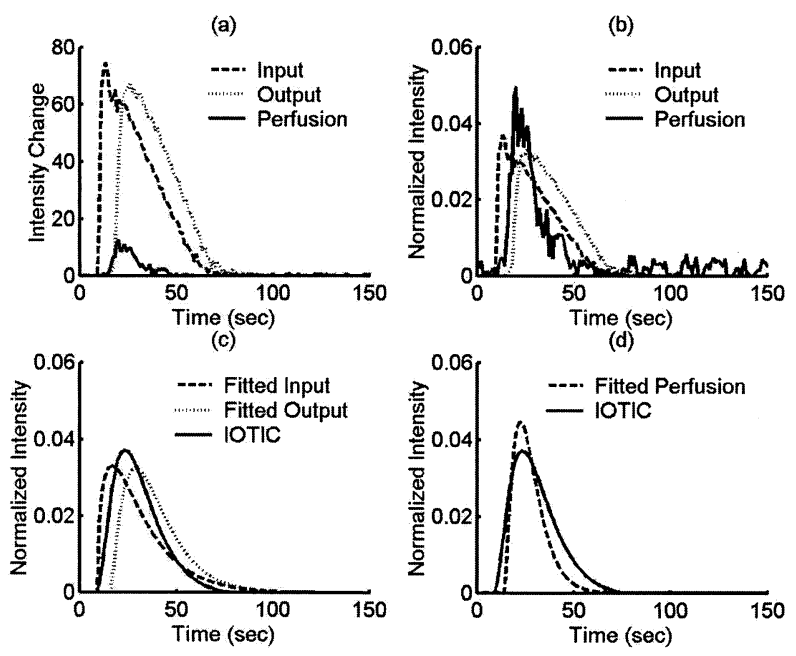

Fig. 7. (a) The input, output and perfused-area TICs obtained from grey-scale images. (b) Normalized TICs; (c) gammafunction-fitted input and output TICs and the derived IOTIC; (d) direct measurement (----) vs. IOTIC (-) .

and solid lines, respectively. Note that the ROI that corresponded to the maximum AUC (as shown in Fig. $5 b)$ was used to obtain the perfusion TIC.

In this study, destruction of microbubbles by the acoustic stimulation was assumed to be negligible, based on the fact that the ratio of the input AUC to the output AUC was close to unity. Figure $7 \mathrm{~b}$ shows the three TICs in Fig. 7a, with the AUC of each curve normalized to unity. After gamma-function fitting, the fitted input and output TICs are shown in Fig. 7c by the dashed and dotted lines, respectively. Finally, the directly measured perfusion TIC (after fitting) and the calculated IOTIC are shown in Fig. 7d by the dashed and solid lines, respectively. The calculated TIC was obtained from eqn (8) using the fitted input and output TICs. Note that the parameters derived from the two curves are likely to be different.

The two TICs, as shown in Fig. 7d, were used to determine two flow parameters as a function of the flow rate: they were MTT and time constant (D'Sa 1999). The results are shown in Figs. 8 and 9. The results of the measured perfusion TICs and the TICs obtained from the IOTIC technique are shown in each figure by the dotted and dashed lines, respectively. Linear regression was performed, and the lines of best fit are shown by the solid lines. The horizontal axis represents the flow rate and the vertical axis indicates to the specific flow parameter. The results shown in Fig. 8 correspond to the estimated MTTs at four flow rates. The correlation coefficients between the estimated MTTs and the best-fit lines were 0.91 and 0.90 , respectively. The high correlation coeffi- 


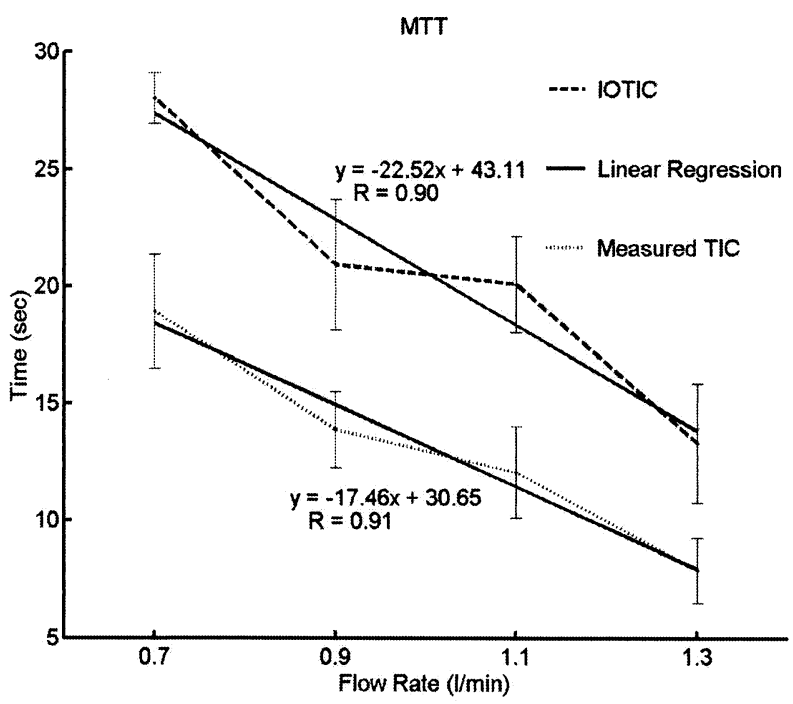

Fig. 8. MTT estimates vs. flow rates. (----) The results of IOTIC, (.....) measured TIC and (-) linear regression.

cients indicate that relative flow measurement is possible with both time-intensity based methods.

The estimated time constants, $\tau$ in eqn (2), from measured TICs and TICs obtained using the IOTIC technique are shown in Fig. 9. The time constant was determined from the best least-squares fit between the TIC and an exponential function. Only the portion of the TIC after the peak intensity was used. The correlation coefficients between the estimated time constants and the best-fit lines are 0.96 for the IOTIC method and 0.63 when derived from the measured TIC.

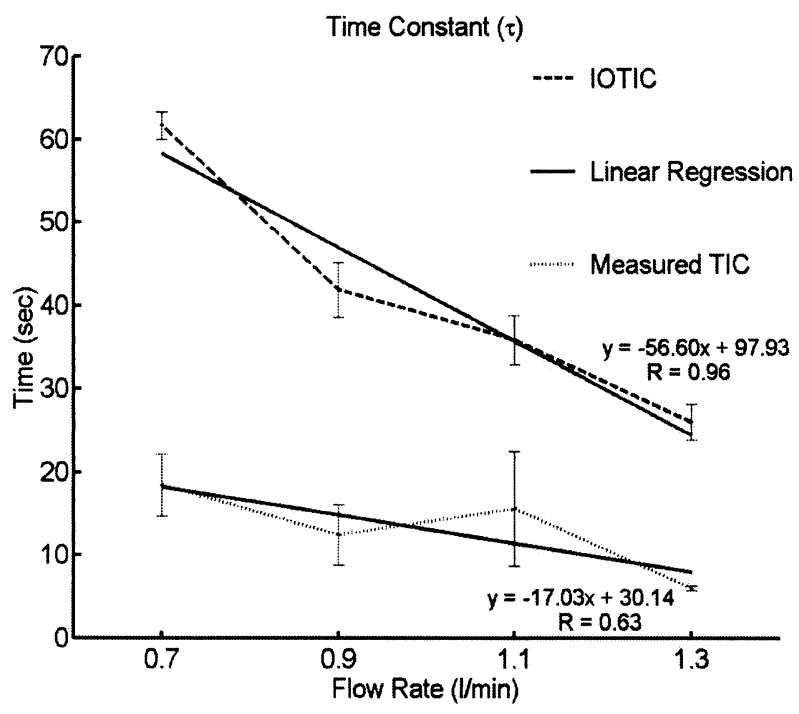

Fig. 9. Time constant vs. flow rates. (----) The results of IOTIC, (.....) measured TIC and (-) linear regression.
The results shown in Figs. 8 and 9 demonstrate that parameters estimated on the basis of the TICs using the IOTIC method generally had higher correlation coefficients with the flow rates than when the directly measured TICs were used. The estimated parameters from measured TICs are all smaller than those obtained by the IOTIC method. Moreover, relative perfusion flow estimation is possible using the IOTIC technique, and this technique only uses the input and output TICs to derive the perfusion parameter and, thus, avoids the shadowing effect.

\section{CONCLUSIONS AND DISCUSSION}

This paper has discussed the shadowing effect that occurs when a contrast agent passes through a perfused region. Because the TIC measured within the perfused area is affected by the shadowing effect, it is not a reliable method for deriving flow parameters. In addition, the proposed technique may be more valuable where the time intensities in the perfused region cannot be reliably measured (e.g., brain). In this case, the TIC in the perfused region can be reconstructed using the input TIC (e.g., carotid artery) and the output TIC (e.g., jugular vein). Certainly, such a long-term research goal can be achieved only after several critical issues have been addressed, and this study simply provides initial results of the entire investigation. One of the critical issues is the correlation between local perfusion conditions and the reconstructed TIC. The other issue is the constant flow rate assumption. Both issues can be addressed by applying proper deconvolution techniques to obtain the transfer function of the dilution process and this is a main focus of current research work.

The shadowing effect depends on the concentration of the contrast agent. In clinical conditions, it may also occur in a heart chamber or a blood vessel (Goldberg 1997). It has been shown that the shadowing effect may limit penetration of the US beam and affect visualization of myocardial layers in contrast echocardiography (Bos et al. 1996; Kaul et al. 1992). The new IOTIC technique monitors the signal enhancement at both the input and the output of a perfused region. Although all the three TICs may potentially be affected, the shadowing effect is less significant on the inflow and the outflow TICs because it can be minimized with a lower microbubble concentration.

It has been shown that several flow parameters obtained from the IOTIC technique are more linearly related to the flow rate than those obtained from the TIC measured directly from the perfused area. These results indicate that the IOTIC technique can replace direct measurement methods. The IOTIC technique provides several advantages: 1 . it does not require assumptions 


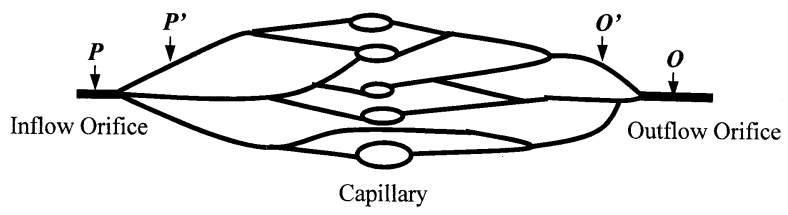

Fig. 10. A schematic diagram of the flow-system model with multiple inputs and multiple outputs.

regarding the input concentration curve, 2. it is less affected by the shadowing effect and 3 . the difficulties associated with gaining acoustic access to certain perfused areas (e.g., brain tissue) can be avoided.

Future work includes the applications of the IOTIC technique to kidneys and extracranial brain perfusion assessment. First, the IOTIC can be applied in renal perfusion assessment by measuring the TICs from the renal artery and the renal vein, assuming that the loss of microbubbles through the urine metabolism can be ignored. The measured perfusion can be used as an index for kidney function after a transplant. Second, the time-intensity relationships can be measured both in a carotid artery and an internal jugular vein to assess brain perfusion. Consider a simple perfusion flow system, shown in Fig. 10, with a single inflow orifice $P$ (e.g., the common carotid artery) and a single outflow orifice $O$ (e.g., the cardinal vein). The inflow orifice $P$ divides into a number of branches, one of which is $P^{\prime}$ (e.g., the internal or external carotid artery) and they continue to divide and terminate at capillaries. Outflow channels coadunate progressively, one of them being represented by $O^{\prime}$ (e.g., the internal jugular vein) and, finally, all venous drainage flows out through the single channel $O$ (Meier and Zierler 1954). The application of the IOTIC method in this case implies measurements of the time intensities from the internal carotid artery and the internal jugular vein, because the two vessels can be monitored simultaneously using conventional grey-scale imaging. Thus, the simple singleinput single-output model adopted in this study needs to be replaced with a multiple-input multiple-output model. Further analysis of multiple-input multiple-output systems is necessary, although, if it is assumed that the concentration curve is essentially the same for all output branches (i.e., the fraction of the total flow leaving through any output channel is proportional to the flow rate in that channel and the shape of the concentration curve is the same for all output branches), eqn (7) will still be valid for flow and volume calculations. The perfusion flow assessments using IOTIC will be correlated with the results from the direct measurement method.

Acknowledgments-Comments from the reviewers and support from the National Health Research Institutes (ROC) (grant NHRI-EX91-
9127EI) are gratefully acknowledged. The authors also thank Dr. W.-C. Yeh for helpful discussion.

\section{REFERENCES}

Bos LJ, Piek JJ, Spaan JA. Effects of shadowing on the time-intensity curves in contrast echocardiography: A phantom study. Ultrasound Med Biol 1996;22:217-227.

Chang PH, Shung KK. Attenuation and backscatter measurements on Albunex. IEEE Ultrason Sympos 1993;2:913-916.

Chen X, Schwarz KQ, Phillips D, Steinmetz SD, Schlief R. A mathematical model for the assessment of hemodynamic parameters using quantitative contrast echocardiography. IEEE Trans Biomed Eng 1998;45:754-765.

Claassen L, Seidel G, Algermissen C. Quantification of flow rates using harmonic grey-scale imaging and an ultrasound contrast agent: an in vitro and in vivo study. Ultrasound Med Biol 2001;27:83-88.

Cooney DO. Biomedical engineering principles: An introduction to fluid, heat and mass transport processes. New York: M. Dekker, 1976.

D'Sa A. Acoustic densitometry-white paper. Hewlett Packard. Boeblingen, Germany: Agilent Technologies, 1999.

Goldberg BB. Ultrasound contrast agents. London: Dunitz Ltd., 1997: 27-29.

Heidenreich PA, Wiencek JG, Zaroff JG, et al. In vitro calculation of flow by use of contrast ultrasonography. J Am Soc Echocardiogr $1993 ; 6: 51-61$

Kaul S, Jayaweera A, Glasheen WP, Villanueva FS, Gutgesell Hp. Myocardial contrast echocardiography and transmural distribution of flow: A critical appraisal during myocardial ischemia not associated with infarction. J Am Coll Cardiol 1992;20:1005-1016.

Li PC, Yeh CK, Wang SW. Time-intensity-based volumetric flow measurements: An in vitro study. Ultrasound Med Biol 2002;28: 349-358.

Meier P, Zierler KL. On the theory of the indicator-dilution method for measurement of blood flow and volume. J Appl Physiol 1954;6: 731-744.

Miles K, Dawson P, Blomley M. Functional computed tomography. Oxford, UK: Isis Medical Media, Oxford University Press, 1997: 49-51.

Mor-Avi V, Akselrod S, David D, Keselbrener L, Bitton Y. Myocardial transit time of the echocardiographic contrast media. Ultrasound Med Biol 1993a;19:635-648.

Mor-Avi V, David D, Akselrod S, Bitton Y, Choshniak I. Myocardial regional blood-flow-Quantitative measurement by computer-analysis of contrast-enhanced echocardiographic images. Ultrasound Med Biol 1993b;19:619-633.

Nanda NC, Schlief R, Goldberg B. Advances in echo imaging using contrast enhancement. Dordrecht, Boston: Kluwer Academic Publishers, 1997.

Postert T, Hoppe P, Federlein J, et al. Contrast agent specific imaging modes for the ultrasonic assessment of parenchymal cerebral echo contrast enhancement. J Cereb Blood Flow Metab 2000;20:17091716.

Schwarz KQ, Bezante GP, Chen X, Mottley JG, Schlief R. Volumetric arterial flow quantification using echo contrast. An in vitro comparison of three ultrasonic intensity methods: radiofrequency, video and Doppler. Ultrasound Med Biol 1993;19:447-460.

Simpson DH, Burns PN, Averkiou MA. Techniques for perfusion imaging with microbubble contrast agents. IEEE Trans Ultrason Ferroelectr Freq Control 2001;48:1483-1494.

Ugolini P, Delouche A, Herment A, Diebold B. In vitro flow quantification with contrast power Doppler imaging. Ultrasound Med Biol 2000;26:113-120.

Wilkening W, Helbeck S, Postert T, et al. Brain perfusion imaging using contrast agent specific imaging modes. IEEE Ultrason Symp 1999;2:1721-1724.

Yeh CK, Wang SW, Li PC. Feasibility study on the time-intensity based blood flow measurements using deconvolution. Ultrason Imaging 2001;23:90-105. 